\title{
${ }^{18}$ F-FDG PET/MR Assessment of Pediatric Langerhans Cell Histiocytosis
}

\author{
Jialing $\mathrm{Niu}^{1,2}$ \\ Jiangtao Liang $^{3}$ \\ Qi Feng ${ }^{2}$ \\ Mei Wang ${ }^{2}$ \\ Luoyu Wang ${ }^{4}$ \\ Xiuhong $\mathrm{Ge}^{2}$ \\ Xiao Wang ${ }^{5}$ \\ Zhongxiang Ding ${ }^{2,6}$
}

'Zhejiang Chinese Medical University, Hangzhou, People's Republic of China;

${ }^{2}$ Department of Radiology, Affiliated Hangzhou First People's Hospital

Zhejiang University School of Medicine, Hangzhou, People's Republic of China;

${ }^{3}$ Hangzhou Universal Medical Imaging

Diagnostic Center, Hangzhou, People's

Republic of China; ${ }^{4}$ Institutes of Psychological Sciences, Hangzhou Normal University, Hangzhou, People's Republic of China; ${ }^{5}$ Department of Radiology, Wenrong Hospital of Hengdian, Jinhua, People's Republic of China;

${ }^{6}$ Translational Medicine Research Center, Key Laboratory of Clinical Cancer Pharmacology and Toxicology Research of Zhejiang Province, Affiliated Hangzhou First People's Hospital, Zhejiang University School of Medicine, Hangzhou, People's Republic of China
Correspondence: Xiao Wang;

Zhongxiang Ding

Email398043951@qq.com;

hangzhoudzx73@I26.com
Introduction: Langerhans cell histiocytosis (LCH) is a histiocytic proliferative disease without a well-understood etiology. The aim of our study is to summarize the imaging features of PET/MR in children with LCH and to explore its diagnostic role in LCH.

Methods: Retrospective analysis was performed of the pretreatment PET/MR imaging data of 15 children with LCH. Comparison of ADC values was done between lesions and normal tissues.

Results: Of the fifteen patients enrolled, five had single-organ or single-system involvement, and ten had multiple-system involvement. Nine patients had varying degrees of bone destruction and increased FDG uptake, whereas thickening and deviation of the pituitary stalk and disappearance of the normal high-signal intensity of T1WI in the neurohypophysis were observed in the pituitary gland in six of them. Splenomegaly with diffuse increased FDG uptake or a normal spleen with increased FDG uptake was found in four cases, liver in three, multiple lymph node enlargement in three, pulmonary lesions in three, and increased metabolism in medullary cavity in two cases. Additionally, two cases involved the skin. Hypermetabolic nodules were detected in muscle in one case, thyroid involvement in one case, and a mediastinal lesion in one case.

Conclusion: PET/MR can show well the distribution of the organs, systems, and lesions involved in LCH and is of considerable significance in the systemic evaluation of LCH.

Keywords: LCH, PET/MR, fluorodeoxyglucose $\mathrm{F}^{18}$, imaging, histiocytosis, eliminate imaging

\section{Introduction}

Langerhans cell histiocytosis (LCH) is a histiocytic proliferative disease without a well understood etiology. Although its etiology is unknown and can occur in all age groups, it is most common in children. According to recent reports, the annual incidence rate of $\mathrm{LCH}$ in children younger than 15 years is $2-9$ cases per million, ${ }^{1}$ Langerhans cells, which originate from the myeloid progenitor cells of the bone marrow, are dendritic cells that can specifically express CD207+ and CD1a+. ${ }^{2}$ Pathology is characterized by abnormal clonal proliferation and aggregation of the Langerhans cells in a single system or multiple systems. ${ }^{3}$ Although LCH can affect all organs of the body, it occurs most frequently in the bones $(80 \%)$, followed in a descending order by the skin (33\%), pituitary gland (25\%), liver (15\%), spleen $(15 \%)$, bone marrow (15\%), lungs $(15 \%)$, lymph nodes $(5-10 \%)$, and the central nervous system $(2-4 \%){ }^{4}$ The disease can be limited to a single system, which is called single-system Langerhans cell histiocytosis (SS-LCH), which is with a good prognosis, or it can affect two or more systems, which is multi-system Langerhans 
cell histiocytosis (MS-LCH). Based on the organs involved, multi-system LCH can also be divided into a low-risk group and a high-risk group. In the high-risk group, the condition involves organs, including the liver, the spleen, and the hematopoietic system, and its prognosis is poor. ${ }^{5,6}$ Therefore, accurate preoperative risk stratification is critical for determining the treatment and prognosis.

Historically, conventional imaging methods have been used for the diagnosis of LCH including radiographic skeletal survey, and ${ }^{99} \mathrm{Tc}-\mathrm{MDP}$ whole-body bone scintigraphy (WBS). Radiographic skeletal survey can show osteolytic destruction and periosteal reaction of the bones, ${ }^{7}$ but it does not display well soft tissue masses, and the sensitivity of radiographic skeletal survey is lower due to the presence of intestinal gas or solid organ occlusion in some specific areas. ${ }^{8}$ Although ${ }^{99}$ Tc-MDP whole-body bone scintillation imaging (WBS) helps to narrow the scope of the differential diagnosis, it cannot be applied for the diagnosis of LCH and is no longer recommended because of the high false-negative rate. ${ }^{9}$ Axial computed tomography (CT) and magnetic resonance imaging (MRI) have been confirmed to be effective in the detection and identification of bone lesions and intracranial involvement in recent years, ${ }^{10,11}$ but they cannot accurately detect active lesions. Whole-body diffusion-weighted MRI (WB-MRI) had higher detectability for LCH lesions than radiographic skeletal survey in primary staging. ${ }^{12}$ But, WB-MRI may be relatively insensitive in the identification of cranial top lesions. The common deficiency of all the abovementioned methods is that they cannot detect the activity of the lesions, and thus may not be the best approach to guide the treatment and evaluate the prognosis. PET/CT is significantly sensitive to skeletal staging and can identify lesions smaller than $10 \mathrm{~mm},{ }^{13}$ but the radiation dose is relatively high. Integrated ${ }^{18} \mathrm{~F}$-FDG PET/MR whole-body imaging is a new multimodal imaging technology which organically combines PET and MRI and has been recently introduced. It collects PET and MRI data at the same time in the same space, and can combine the metabolic information of a radioactive tracer in the PET system with the accurate anatomical data of MRI. In clinical work, PET/ MR can improve the accuracy of diagnosis of many diseases. Recently, Feng found that PET/MR had a high diagnostic value for clinical staging of nasopharyngeal carcinoma. ${ }^{14}$ Paspulati's Study has shown that PET/MR had clinical utility value in the follow-up evaluation of gastrointestinal tumors after treatment. ${ }^{15}$ In addition, other study has also confirmed the high diagnostic significance of PET/MR in pediatric oncology. ${ }^{16}$ PET/MR imaging is a promising method whose radiation exposure is essentially lower than that of PET/CT; it also has great potential in the diagnosis of LCH in children. In the present study, we performed retrospective analysis of the PET/MR imaging data of 15 children with LCH. In this article, we provide analysis, insights, summarize the characteristics of PET/MRI imaging in children with LCH, and discuss the value of PET/MR in assessing the involvement of systems and organs in children with LCH.

\section{Methods}

\section{Patients}

A retrospective analysis was performed on 15 children with LCH who were examined at Hangzhou Universal Medical Imaging Diagnostic Center from April 2017 to July 2019. The age of the selected subjects, including seven males and eight females, ranged from 22 months to 9 years, with an average age of $4.17 \pm 3.42$ years. All the fifteen patients performed PET/MR examination at the first diagnosis without any form of treatment, except one patient underwent sphenoidectomy before the examination and was confirmed as LCH by postoperative pathology. One patient was confirmed as LCH by skin biopsy before the examination, and the remaining thirteen patients were determined by surgery or biopsy pathology after the examination. All patients' legal guardians signed a written informed consent form before the examination. This retrospective study involving human participants was reviewed and approved by the Hangzhou Universal Medical Imaging Diagnostic Center Medical Ethics Committee (No. 202101). Our study was conducted in accordance with the Declaration of Helsinki.

\section{PET/MR Scans Acquisition}

The following preparation before the examination was done. The patient fasted for more than $6 \mathrm{~h}$, with a blood glucose concentration that had to be maintained below 7.8 $\mathrm{mmol} / \mathrm{L}$ before the injection of ${ }^{18} \mathrm{~F}$-FDG. The patients were injected with ${ }^{18} \mathrm{~F}-\mathrm{FDG}$ at a dose of $3.7 \mathrm{Mbq} / \mathrm{kg}$ and placed in the supine position. Then, whole-body PET/MR scanning was performed after $40 \mathrm{~min}$. In children who were unable to cooperate, we used $10 \%$ chloral hydrate oral or enema for sedation, or hypnosis 20-30 min before the start. All patients also performed high-resolution $\mathrm{CT}$ examination of the lung. 


\section{PET/MR Imaging}

After performing the attenuation correction, performed a whole-body PET/MR scan from the top of the head to the middle of the femur and scanned to the bottom of the feet if necessary for a total of 5-6 beds. The collection time for each bed was 6 minutes. Whole-body imaging was performed in the supine position with an integrated SIGNA PET/MR 3.0T system (GE SIGNA, Wisconsin, USA). The MR sequence included axial LAVA-Flex T1, fs-PROPELLER T2, DWI ( $b=800 \mathrm{~s} / \mathrm{mm} 2)$, slice thickness $6.0 \mathrm{~mm}$, slice spacing $1.0 \mathrm{~mm}$, FOV $(34.0 \mathrm{~cm})$, coronal fs-PROPELLER T2, slice thickness $5.5 \mathrm{~mm}$, slice spacing $1.0 \mathrm{~mm}$, FOV $(42.0 \mathrm{~cm})$. PET/MR examination was performed within 40-60 min after the injection of the imaging agent. The examination time was approximately $40 \mathrm{~min}$, after which the respiratory gating was collected. Meanwhile, PET images were collected in the $3 \mathrm{D}$ mode using OSEM and TOF information as the reconstruction method, and PSF correction.

\section{Image Analysis}

Image analysis was conducted using a GE workstation (AW Server 2.0). The maximum standardized uptake value (SUVmax) was measured by an experienced nuclear medicine physician using semi-quantitative methods. According to the signal of the focus and the ${ }^{18} \mathrm{~F}-\mathrm{FDG}$ metabolism, the organs and systems involved in LCH were determined, and the region of interest (ROI) was delineated. The SUVmax of the region of interest on PET/MR images was measured and the radioactivity background of normal liver parenchyma was established in each case. The data of the location, size, shape, signal, and ${ }^{18} \mathrm{~F}$-FDG metabolism of all lesions were recorded. Diagnostic criteria for $\mathrm{LCH}$ positive lesions: a lesion was judged positive if it showed non-physiological ${ }^{18} \mathrm{~F}$-FDG uptake that was higher than the mean uptake of the patient's right liver lobe. Spleen and liver were considered positive if they were increased in size, multiple nodules in parenchyma, or the liver's Glisson sheath widened with irregular margins. Lymph nodes were considered positive if their short diameter $\geq 10 \mathrm{~mm}$. The apparent diffusion coefficient (ADC) map was reconstructed on the postprocessing workstation. The ROI was placed inside the outer margin of the largest lesion on the ADC map to reduce partial volume effect interference. ADC values of lesions and normal tissue were obtained, including the
Table I Distribution of the Number of Organs Involved

\begin{tabular}{|l|c|c|c|}
\hline & SS-LCH(n) & MS-LCH(n) & Total(n) \\
\hline Bone & 3 & 6 & 9 \\
Pituitary gland & $\mathrm{I}$ & 5 & 6 \\
Spleen & 0 & 4 & 4 \\
Liver & 0 & 3 & 3 \\
Lymph nodes & 0 & 3 & 3 \\
Lung & 0 & 3 & 3 \\
Bone marrow & 0 & 2 & 2 \\
Muscle & 0 & 1 & 1 \\
Skin & 1 & 1 & 2 \\
Thyroid gland & 0 & 1 & 1 \\
\hline
\end{tabular}

minimum $\mathrm{ADC}$ value (ADCmin), maximum $\mathrm{ADC}$ value (ADCmax) and average ADC value (ADCave).

\section{Statistical Analysis}

Statistical analyses were performed with SPSS version 22.0. The rank sum test (in accordance with skewed distribution) was used to compare ADCave values of the lesions with those of normal tissues. $P<0.05$ was statistically significant.

\section{Results}

Of the fifteen patients, five had single-organ or singlesystem involvement (three in the bone, one in the pituitary gland, and one in the skin) and ten multiple systems. In all cases, the skeletal system was most affected (nine cases), followed by the pituitary gland (six cases), the spleen (four cases), the liver (three cases), the lymph nodes (three cases), the lungs (three cases), the bone marrow (two cases), the skin (two cases), the muscle (one case), the thyroid (one case), and the mediastinum (one case). All affected organs and systems are listed in Tables 1 and 2 .

\section{Skeletal System Craniofacial Bone}

The craniofacial bones of the four patients were involved, including temporal bone, mastoid, sphenoid, occipital bone, cavernous sinus, maxilla, clivus, and hard palate. PET/MR showed local osteolytic bone destruction, soft tissue mass formation, unclear mass boundary, iso-or slightly low-signal intensity of T1WI, iso-or low-signal intensity of T2WI, limited diffusion of DWI, and an increased uptake of FDG in different degrees. The average SUVmax established was $4.8 \pm 3.6$ (Figure1). 
Table 2 Patient Characteristics, Distribution of System/Organ Involved and Site of Histological Examination

\begin{tabular}{|c|c|c|c|c|c|}
\hline $\begin{array}{l}\text { Patient } \\
\text { No. }\end{array}$ & Gender & Age & $\begin{array}{l}\text { System } \\
\text { Involvement }\end{array}$ & Distribution of System/Organ Involved & $\begin{array}{l}\text { Site of Histological } \\
\text { Examination }\end{array}$ \\
\hline 1 & M & $4 y$ & MS-LCH & Spine, spleen & Vertebrae \\
\hline 2 & $\mathrm{~F}$ & $6 y$ & SS-LCH & Limb bones & Ilium \\
\hline 3 & $\mathrm{~F}$ & $8 y$ & MS-LCH & Pelvis, lung & Sacrum \\
\hline 4 & M & $5 y$ & MS-LCH & Pituitary gland, lymph node & Lymph node \\
\hline 5 & M & $22 m$ & MS-LCH & Mediastinum, liver & Mediastinum \\
\hline 6 & M & $2 y$ & MS-LCH & Skeletal system, pituitary gland, lung, spleen & Sacrum \\
\hline 7 & M & $7 y$ & SS-LCH & Craniofacial bone & Sphenoid bone \\
\hline 8 & $\mathrm{~F}$ & $4 y$ & MS-LCH & Muscle, skin & Skin \\
\hline 9 & $\mathrm{~F}$ & $8 y$ & MS-LCH & $\begin{array}{l}\text { Pituitary gland, thyroid, spleen, bone marrow, lymph } \\
\text { nodes, rib }\end{array}$ & Lymph node \\
\hline 10 & M & $8 y$ & MS-LCH & Pituitary gland, skeletal system, lymph node & Ilium \\
\hline II & $\mathrm{F}$ & $2 y$ & SS-LCH & Skeletal system, bone marrow & Ilium \\
\hline 12 & $\mathrm{~F}$ & $9 y$ & MS-LCH & Pituitary gland, lung, lymph node & Lymph node \\
\hline 13 & $\mathrm{~F}$ & ly & MS-LCH & Skeletal system, spleen & Temporal bone \\
\hline 14 & M & ly & SS-LCH & Skin & Skin \\
\hline 15 & $\mathrm{~F}$ & $9 y$ & SS-LCH & Pituitary gland & Pituitary gland \\
\hline
\end{tabular}

\section{Limbs}

Bone involvement of extremities was found in three patients, which showed local cyst bone destruction and defect, abnormal signal intensity, high-signal intensity on the fat compression image, some of which accompanied by peripheral soft tissue exudation and edema, and increased metabolism of FDG in different degrees. The average SUVmax was $2.8 \pm 0.8$.

\section{Spine}

Vertebral involvement was detected in three patients, showing compression wedge degeneration of the vertebral body, "flat vertebra", uneven signal of the vertebral body, slightly longer $\mathrm{T} 1$ and long $\mathrm{T} 2$ signal changes, bone destruction of the vertebral body and pedicle, with a local soft-tissue shadow, increased FDG uptake. The average SUVmax was $4.9 \pm 5.5$.

\section{Pelvic Constituent Bone}

Hip/sacrum/ilium/ischium involvement was found in five cases, which showed local osteolytic bone destruction and partial bone substance marginal hardens. Multiple abnormal signals and irregular patchy shadow could be seen in some of the involved bones. T1WI showed low or slightly low-signal intensity, T2WI showed high-signal intensity. DWI was clearly diffused and limited, with or without soft tissue signal mass, some with residual bone. FDG metabolism was increased; the average SUVmax was $6.4 \pm 7.5$ (Figure 2C-F).

\section{Ribs}

One patient had rib involvement, associated with bone destruction, soft tissue density shadow, and increased FDG uptake. The SUVmax value was 7.98.

\section{Pituitary Gland}

Pituitary involvement was found in six patients, with thickening of the pituitary stalk with deviation. One patient had abnormal signals near the pituitary stalk, involving the pituitary stalk and para-hypothalamic nodules, and one patient had small nodules behind the optic chiasm. The absence of the normal high-signal intensity of T1WI was observed in all 6 patients and an increase in FDG metabolism in pituitary gland. The average SUVmax was $3.1 \pm 0.7$. 
A

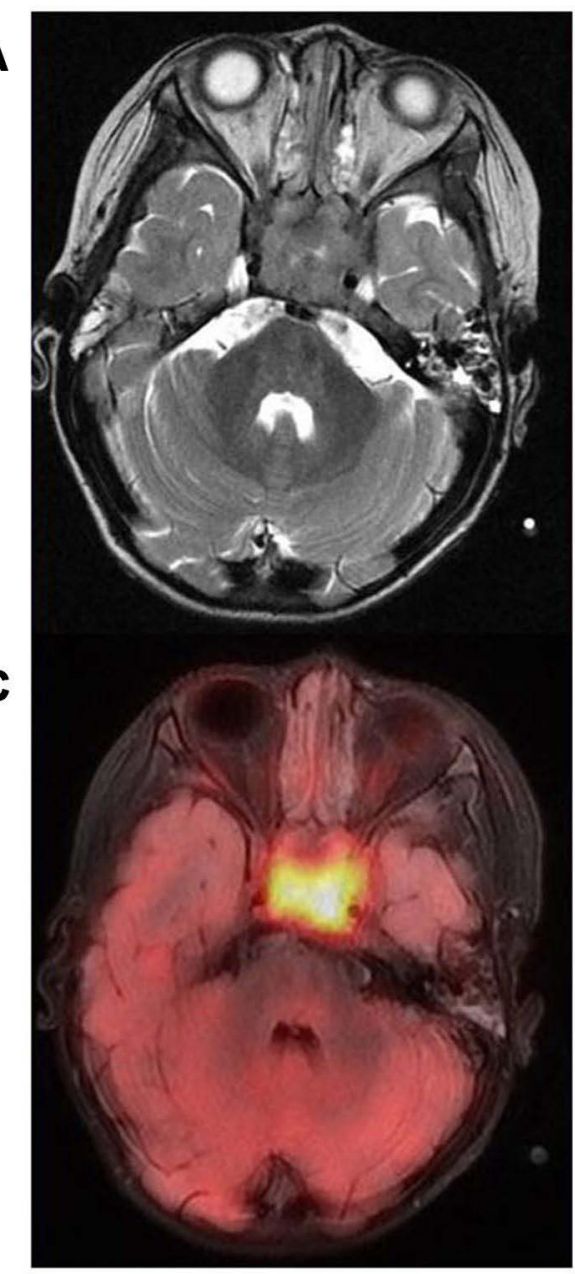

B

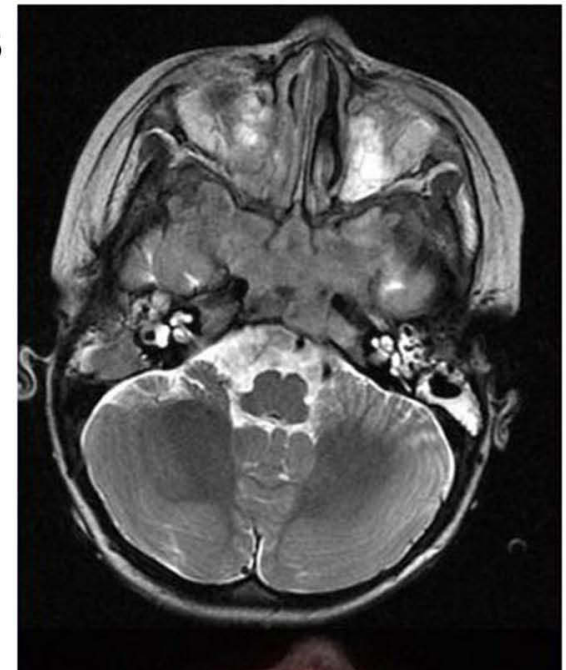

D

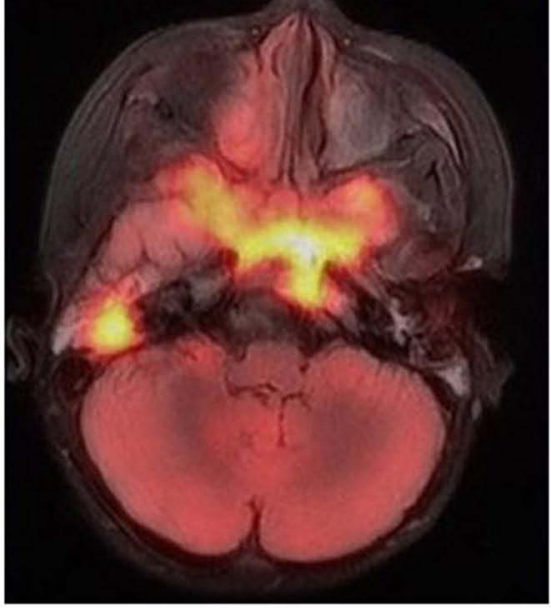

Figure I Multi-system LCH child (male, 2 years old) with bone destruction of sphenoid body, disappearance of normal sphenoid sinus cavity, irregular soft tissue mass, increased FDG uptake, invasion of bilateral cavernous sinus, temporal fossa, right nasal cavity, maxillary sinus and maxilla (A-D). (upper rows: T2-weighted images; lower rows: PET/MR images).

\section{Liver and Spleen}

Three patients were with liver involvement. One patient with multiple nodules in the liver parenchyma, limited diffusion of DWI sequence, and increased FDG metabolism. The SUVmax was 5.51. Two patients had an enlarged liver volume. The spleen was involved in four cases, three of which showed enlargement of the spleen and a diffuse increase in FDG uptake; normal spleen size and increased FDG uptake was established in one case. The average SUVmax was $2.0 \pm 0.9$.

\section{Lymph Nodes}

The lymph nodes were affected in three patients. The main manifestations were multiple enlargements of the lymph nodes in the bilateral neck, the submandibular, submental, and cervical posterior triangle, and the supraclavicular region, as well as increased FDG metabolism. The average SUVmax was $5.2 \pm 1.5$.

\section{Other Manifestations}

Pulmonary lesions occurred in three patients, which were mainly manifested as nodular foci or patchy blurred shadow in the lungs, increased FDG in two patients. The average SUVmax was 5.6 3.6. And background uptake of FDG in another patient (Figure 2A and B). A slight increase in FDG metabolism in multiple medullary cavities in two patients. The average SUVmax was $4.2 \pm 2.4$. Hypermetabolic nodules were found in the deltoid muscle and the proximal lateral muscle of the left thigh in one patient. The SUVmax was 3.28. This patient was accompanied by skin rashes. One 


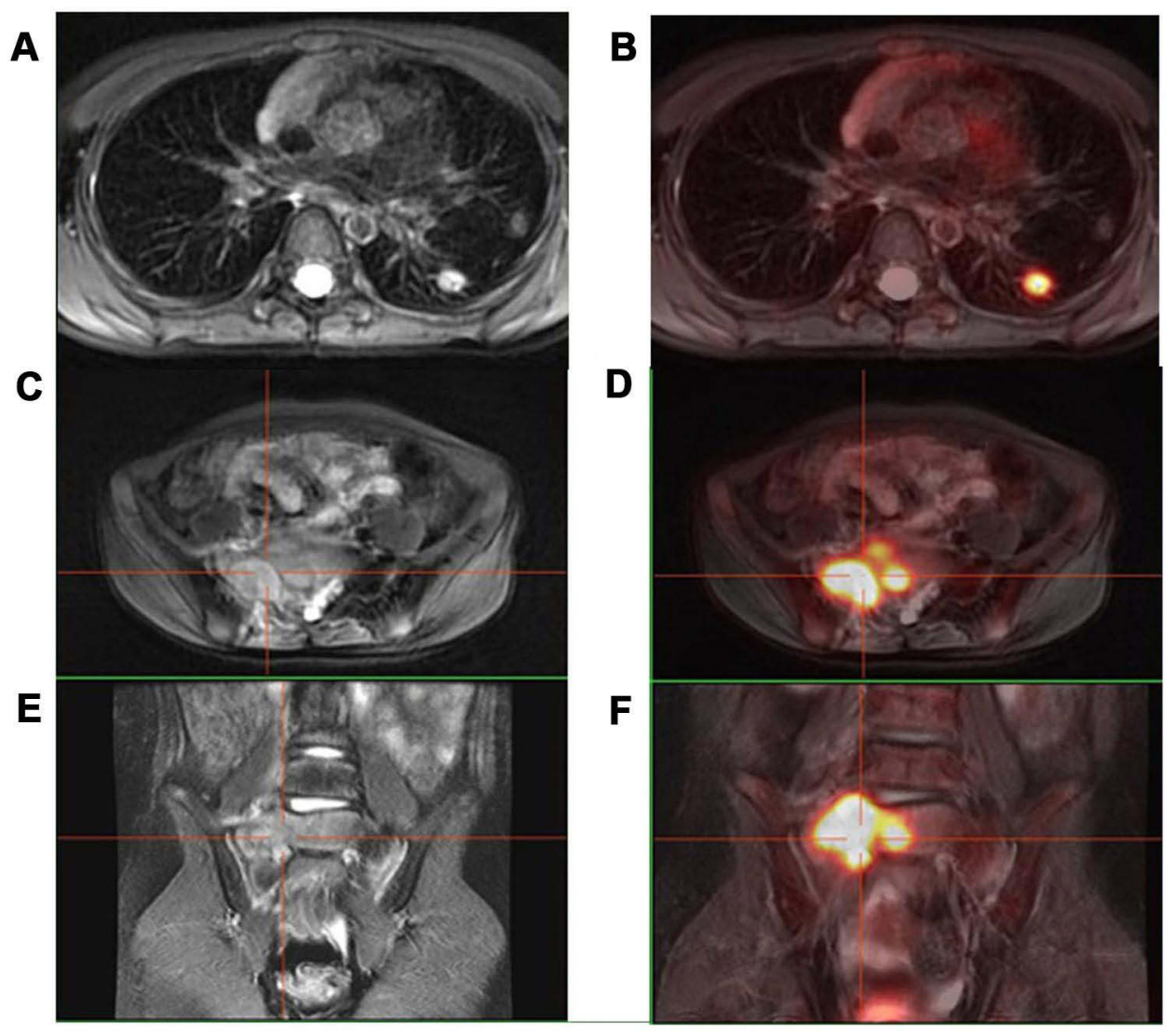

Figure 2 Multiple system LCH in children (female, 8 years old) with nodular lesions in the left lower lobe of the lung, increased FDG metabolism (A and B), and right sacral soft tissue density mass bone destruction, FDG metabolism increased (C-F). (left columns: T2-weighted images; right columns: PET/MR images).

case had a large irregular mass in the anterior superior mediastinum with FDG hypermetabolism. The SUVmax value was 8.19. A slight increase in FDG metabolism was detected in multiple locations on the skin of the whole body in one patient. The SUVmax was 0.36. The thyroid gland was involved in one patient, and a hypermetabolic nodule was observed in the right lobe of this patient's thyroid gland. The SUVmax was 5.62.

\section{ADC Values}

A total of fifteen patients were included in this study. As some of them had smaller lesions and lesions close to air and bone, which resulted in obvious image artifacts, six cases were finally excluded and a total of nine cases were measured. Among 9 patients, ADCave values of lesions were higher than those of normal tissues in 6 patients and lower than those of normal tissues in 3 patients. The results of rank sum test were $P=0.508$, mean rank of ADCave value of the lesions was $10.33 \mathrm{~mm}^{2} / \mathrm{s}$ and mean rank of ADCave value of the normal tissue was $8.67 \mathrm{~mm}^{2} / \mathrm{s}$.

\section{Discussion}

Through retrospective analysis, we investigated the manifestations of PET/MR in a group of children with LCH and summarized the imaging manifestations of LCH in PET/ MR findings. The results showed various manifestations of LCH. Based on the different manifestations of the involved sites, PET/MR could well display the involved organs and systems, having a significant value in the prognosis evaluation, and treatment of LCH.

The progression and prognosis of LCH vary considerably according to the classification. Mild cases can be selfcured, whereas severe cases may show diffuse involvement of multiple systems and rapid disease progression. Therefore, a different treatment plan is also to be implemented in each specific case. Usually, the condition of patients with single-site involvement can be monitored by local hormone levels or surgically, whereas patients with single-system multi-site or multi-system involvement require the application of chemotherapy combined with local radiotherapy, and the prognosis is poor. ${ }^{17}$ Compared with bone scan and conventional radiography, ${ }^{18}$ F-FDG 
PET has more advantages for the identification of wholebody lesions. It has high sensitivity and specificity in the diagnosis of pediatric $\mathrm{LCH}$, and its use in following the evaluation of $\mathrm{LCH}$ has been fully confirmed, ${ }^{10}$ but clear anatomical evidence is still lacking. MRI can provide more accurate anatomical information, and the overall sensitivity of MRI is better than that of PET. ${ }^{18} \mathrm{~A}$ previous study analyzed PET and MRI images together. ${ }^{18}$ Its results revealed that the method of joint analysis reduced the falsenegative rate of the initial staging of $\mathrm{LCH}$, improved the sensitivity and diagnostic accuracy of LCH but PET/MR device used in the study was a sequential scanner that collects PET and MR data at different time points. The integrated ${ }^{18} \mathrm{~F}$-FDG $\mathrm{PET} / \mathrm{MR}$ technique applied in our study could scan the whole body at once. It evaluated not only the anatomical site of the lesion but also the metabolic activity of the lesion ( ${ }^{18} \mathrm{~F}$-FDG uptake). Therefore, the examination time could be significantly shortened, and image information was collected at the same place and time. In clinical work, PET/MR can clearly show the involvement of various organs and systems, and can identify cells with metabolic activity, which is helpful for the detection of hidden lesions. In this study, the liver was involved in three cases, the spleen in four cases, and the medullary cavity in two cases, suggesting that PET/MR can provide imaging basis for clinical classification and risk classification. Another study confirmed that PET/MR was a feasible alternative to PET/CT. ${ }^{19}$ Compared with PET/CT subjects, who do not need additional radiation exposure, the radiation dose received by the application of PET/MR is significantly reduced, which is of great significance for radiationsensitive children.

Luna et $\mathrm{al}^{20}$ observed the changes of ADC values in brain lumps of 23 patients with histiocytosis. The results showed that ADC values were elevated or normal compared with normal tissues. In our study, we compared the ADC values between lesions and normal tissues. The results showed no statistical difference, possibly due to the small number of cases, but the mean ADC values of the lesions were higher than those of normal tissues. The lack of a significant reduction in ADC values in most patients with LCH may provide some clues for the differential diagnosis of other tumors, such as lymphoma. ${ }^{21}$

The skeletal system is the most frequently involved system in LCH. Most of the images show bone destruction with soft tissue mass formation, surrounding soft tissue exudation and edema, and compression of the adjacent spinal cord. Flat bone is commonly involved, in which skull LCH is most frequent, followed by the long bone and spine, whereas hand and foot short bone involvement is rare. ${ }^{22}$ The vertebrae involved in LCH in our study were widely destroyed; they collapsed and were wedge-shaped, showing the appearance of "flat vertebrae", which was also accompanied by the formation of paraspinal soft tissue. Nine patients had varying degrees of bone destruction. PET/MR not only visualized systemic bone destruction and the soft tissue mass but also facilitated the judgment of the lesion activity by FDG metabolism. FDG uptake increased to varying degrees in all cases in this group.

The imaging diagnosis of pituitary involvement should be combined with clinical diagnosis. Patients usually have a history of diabetes insipidus. Imaging findings generally show thickening of the pituitary stalk ( $>3 \mathrm{~mm}$ ), increased FDG uptake. The normal high-signal intensity of neurohypophysis disappeared on T1WI images. The adjacent hypothalamus, optic chiasma and third ventricle can also be involved. Because the neurosecretory granules containing antidiuretic hormone are stored in the posterior pituitary, the normal posterior pituitary showed high-signal intensity on the T1WI images. When LCH involves the pituitary, it destroys the neuropituitary channel, resulting in inability for transport of the antidiuretic hormone neurosecretory granules into the posterior pituitary region. Then, the posterior pituitary hypersignal disappears and the neurological function of the pituitary is disordered or damaged, which is clinically manifested as diabetes insipidus. Therefore, the existence of a high signal is a sign to judge whether the neurological function of the pituitary gland is normal. In this respect, PET/MR can well show the involvement of the pituitary gland and the storage function of neurohypophysis.

Pulmonary LCH (PLCH) is a chronic progressive interstitial lung disease, which is divided into two types. One of them involves the lungs alone, which is called primary $\mathrm{PLCH}$, also known as isolated $\mathrm{PLCH}$, which is common in adults and is related to smoking. The second type of PLCH is part of multi-system $\mathrm{LCH}$, commonly referred to as lung involvement, which is common in children and accounts for $12-50 \%$ of children's multi-system $\mathrm{LCH}^{23}$ The typical imaging findings of PLCH are multiple solid nodules or reticular dense shadows in the early lungs, which are usually bilaterally symmetrical and diffusely distributed. ${ }^{24}$ With the progress of the disease, a cavity may appear, and, with the enlargement of the cavity, the nodules can be transformed into thick-walled and thin-walled cysts, finally presenting grid-like fibrous changes; coexistence of multiple lesions is also observed in some cases. In our study, three children had pulmonary lesions, which were mainly characterized by 
small pulmonary nodules or patchy blurred shadows, and increased FDG or background uptake. The reason for the mild lung manifestation and low metabolism in this group of patients may be that all the children in this study were newly diagnosed, and most of them were in the early stage of the disease, with still mild lung involvement.

$\mathrm{LCH}$ tends to involve organs with a rich reticular system, such as the liver, spleen, lymph nodes. Approximately 51.9\% of the children with $\mathrm{LCH}$ had invasion of the hepatobiliary system, of which $71.4 \%$ occurred in patients with multiplesystem involvement. ${ }^{25}$ The pathological process of liver involvement can be divided into four stages: histiocytic, granuloma, xanthomatous, and fibrotic stages. The corresponding imaging manifestations are also different in these periods. ${ }^{26}$ Generally, hepatomegaly found on images indicates the initial manifestation of liver damage. In the present investigation, three children had intrahepatic lesions, which were mainly characterized by enlarged liver volume, multiple nodules in the liver parenchyma, and increased FDG metabolism. Different MRI findings and FDG metabolic changes reflected different stages of the lesions. Lymph node involvement is usually one of the manifestations of multi-system $\mathrm{LCH}$, and the most frequently involved sites reported in the literature are the neck, armpit, groin, and supraclavicular lymph nodes. ${ }^{27}$ In our study, three children had lymphadenopathy, which was manifested with multiple enlargements of the lymph nodes in the bilateral neck, subjaw, subchin, posterior triangle of the neck, and the supraclavicular region, accompanied by increased FDG metabolism. However, the lymph node LCH was not specific and needed to be distinguished from other diseases that could cause lymph node enlargement.

This study has some limitations due to the low incidence of LCH. Thus, the number of subjects included was limited, and we will further expand the sample size in the future. In addition, the value of PET/MR examination is the detection of active metabolic lesions but for precise assessment of brain, pituitary gland, bones, lung and liver, specific examination should be performed. All patients in our study underwent chest CT examination because of MRI or PET are not sufficient for lung evaluation. MRI enhancement examination has its unique advantages in brain, pituitary gland, and liver. In the future, further research can try to combine PET/ MR and MRI enhancement to obtain more information.

\section{Conclusions}

In short, LCH shows a variety of manifestations, including only bone destruction, single-organ involvement, or multisystem lesions, symptoms related to multiple disciplines and easy to miss diagnosis or misdiagnosis. ${ }^{18}$ F-FDG PET/MR can show better than conventional techniques the involvement of various organ systems and the distribution of lesions. In the future, PET/MR may become the preferred examination method for preliminary staging and post-treatment evaluation of LCH. This approach can also to reduce the examination time and radiation exposure while maintaining a high diagnostic accuracy.

\section{Funding}

The National Natural Science Foundation of China (no. 81871337), the Zhejiang Provincial Medical and Health Technology Project (no. 2020RC092), and the Medical and Health Technology Project of Hangzhou (no. A20200507).

\section{Disclosure}

The authors report no conflicts of interest in this work.

\section{References}

1. Poompuen S, Chaiyarit J, Techasatian L. Diverse cutaneous manifestation of Langerhans cell histiocytosis: a 10-year retrospective cohort study. Eur J Pediatr. 2019;178(5):771-776. doi:10.1007/s00431-01903356-1

2. Monsereenusorn C, Rodriguez-Galindo C. Clinical characteristics and treatment of Langerhans cell histiocytosis. Hematol Oncol Clin North Am. 2015;29(5):853-873. doi:10.1016/j.hoc.2015.06.005

3. Chu T, Jaffe R. The normal Langerhans cell and the LCH cell. $\mathrm{Br}$ J Cancer Suppl. 1994;23:S4-S10.

4. Papo M, Cohen-Aubart F, Trefond L, et al. Systemic histiocytosis (Langerhans cell histiocytosis, Erdheim-Chester disease, Destombes-Rosai-Dorfman disease): from oncogenic mutations to inflammatory disorders. Curr Oncol Rep. 2019;21(7):62. doi:10.1007/s11912-019-0810-6

5. Weitzman S, Egeler RM. Langerhans cell histiocytosis: update for the pediatrician. Curr Opin Pediatr. 2008;20(1):23-29. doi:10.1097/ MOP.0b013e3282f45ba4

6. Heritier S, Emile JF, Barkaoui MA, et al. BRAF mutation correlates with high-risk Langerhans cell histiocytosis and increased resistance to first-line therapy. $J$ Clin Oncol. 2016;34(25):3023-3030. doi:10.1200/JCO.2015.65.9508

7. Zhang X, Zhou J, Chai X, et al. The application of X-ray, computed tomography, and magnetic resonance imaging on 22 pediatric Langerhans cell histiocytosis patients with long bone involvement: a retrospective analysis. Medicine. 2018;97(17):e0411. doi:10.1097/ MD.0000000000010411

8. Girschikofsky M, Arico M, Castillo D, et al. Management of adult patients with Langerhans cell histiocytosis: recommendations from an expert panel on behalf of Euro-Histio-Net. Orphanet $J$ Rare Dis. 2013;8(1):72. doi:10.1186/1750-1172-8-72

9. Van Nieuwenhuyse J-P, Clapuyt P, Malghem J, et al. Radiographic skeletal survey and radionuclide bone scan in Langerhans cell histiocytosis of bone. Pediatr Radiol. 1996;26(10):734-738. doi:10.1007/ BF01383393

10. Phillips M, Allen C, Gerson P, McClain K. Comparison of FDG-PET scans to conventional radiography and bone scans in management of Langerhans cell histiocytosis. Pediatr Blood Cancer. 2009;52 (1):97-101. doi:10.1002/pbc.21782 
11. Grois N, Fahrner B, Arceci RJ, et al. Central nervous system disease in Langerhans cell histiocytosis. J Pediatr. 2010;156(6):873-881 e1. doi:10.1016/j.jpeds.2010.03.001

12. Kim JR, Yoon HM, Jung AY, Cho YA, Seo JJ, Lee JS. Comparison of whole-body MRI, bone scan, and radiographic skeletal survey for lesion detection and risk stratification of Langerhans cell histiocytosis. Sci Rep. 2019;9(1):317. doi:10.1038/s41598-01836501-1

13. Zhou W, Wu H, Han Y, Wang S, Dong Y, Wang Q. Preliminary study on the evaluation of Langerhans cell histiocytosis using F-18-fluorodeoxy-glucose PET/CT. Chin Med J. 2014;127(13):2458-2462.

14. Feng Q, Liang J, Wang L, et al. Radiomics analysis and correlation with metabolic parameters in nasopharyngeal carcinoma based on PET/MR imaging. Front Oncol. 2020;10:1619. doi:10.3389/ fonc.2020.01619

15. Paspulati RM, Gupta A. PET/MR imaging in cancers of the gastrointestinal tract. PET Clin. 2016;11(4):403-423. doi:10.1016/j. cpet.2016.05.004

16. Schafer JF, Gatidis S, Schmidt H, et al. Simultaneous whole-body $\mathrm{PET} / \mathrm{MR}$ imaging in comparison to $\mathrm{PET} / \mathrm{CT}$ in pediatric oncology: initial results. Radiology. 2014;273(1):220-231. doi:10.1148/ radiol.14131732

17. DiCaprio MR, Roberts TT. Diagnosis and management of Langerhans cell histiocytosis. J Am Acad Orthop Surg. 2014;22 (10):643-652. doi:10.5435/JAAOS-22-10-643

18. Mueller WP, Melzer HI, Schmid I, Coppenrath E, Bartenstein P, Pfluger T. The diagnostic value of $18 \mathrm{~F}-\mathrm{FDG}$ PET and MRI in paediatric histiocytosis. Eur J Nucl Med Mol Imaging. 2013;40 (3):356-363. doi:10.1007/s00259-012-2278-6

19. Sher AC, Orth R, McClain K, Allen C, Hayatghaibi S, Seghers V. $\mathrm{PET} / \mathrm{MR}$ in the assessment of pediatric histiocytoses: a comparison to PET/CT. Clin Nucl Med. 2017;42(8):582-588. doi:10.1097/ RLU.0000000000001717
20. Luna LP, Drier A, Aygun N, et al. MRI features of intra-axial histiocytic brain mass lesions. Clin Radiol. 2021;76(2):159e19-159 e28. doi:10.1016/j.crad.2020.09.015

21. Shapira-Zaltsberg G, Wilson N, Trejo Perez E, et al. Whole-body diffusion-weighted MRI compared to 18 F FDG PET/CT in initial staging and therapy response assessment of Hodgkin lymphoma in pediatric patients. Can Assoc Radiol J. 2020;71(2):217-225. doi:10.1177/0846537119888380

22. Ginat DT, Johnson DN, Cipriani NA. Langerhans cell histiocytosis of the temporal bone. Head Neck Pathol. 2016;10(2):209-212. doi:10.1007/s12105-015-0629-x

23. Seely JM, Salahudeen S Sr, Cadaval-Goncalves AT, et al. Pulmonary Langerhans cell histiocytosis: a comparative study of computed tomography in children and adults. J Thorac Imaging. 2012;27 (1):65-70. doi:10.1097/RTI.0b013e3181f49eb6

24. Smets A, Mortele K, de Praeter G, Francois O, Benoit Y, Kunnen M Pulmonary and mediastinal lesions in children with Langerhans cell histiocytosis. Pediatr Radiol. 1997;27(11):873-876. doi:10.1007/ s002470050260

25. Yi X, Han T, Zai H, Long X, Wang X, Li W. Liver involvement of Langerhans' cell histiocytosis in children. Int J Clin Exp Med. 2015;8 (5):7098-7106.

26. Shi Y, Qiao Z, Xia C, et al. Hepatic involvement of Langerhans cell histiocytosis in children-imaging findings of computed tomography, magnetic resonance imaging and magnetic resonance cholangiopancreatography. Pediatr Radiol. 2014;44(6):713-718. doi:10.1007/ s00247-014-2886-3

27. Schmidt S, Eich G, Geoffray A, et al. Extraosseous Langerhans cell histiocytosis in children. Radiographics. 2008;28(3):707-26;quiz 910-1. doi:10.1148/rg.283075108
International Journal of General Medicine

\section{Publish your work in this journal}

The International Journal of General Medicine is an international, peer-reviewed open-access journal that focuses on general and internal medicine, pathogenesis, epidemiology, diagnosis, monitoring and treatment protocols. The journal is characterized by the rapid reporting of reviews, original research and clinical studies across all disease areas. The manuscript management system is completely online and includes a very quick and fair peer-review system, which is all easy to use. Visit http://www.dovepress.com/ testimonials.php to read real quotes from published authors. 